\title{
SELECTED EXAMPLES OF APPLICATIONS OF NEW GRAPHICS AND ANIMATION TECHNOLOGIES
}

\author{
Zbigniew Filutowicz, Krzysztof Przybyszewski’ Józef Paszkowski \\ IT Institute, University of Social Sciences, Łódź, Poland \\ (zfilutowicz,kprzybyszewski)@spoleczna.pl
}

\begin{abstract}
In recent years there has been a marked increase in the competitiveness of some very interesting (user) applications software within the field of computer graphics and animation. This paper presents an analysis of selected examples of the use of graphic applications software designed for professional use within various areas of human activity, and also focusses on the potential for further development of this software. Graphic applications software that makes use of motion capture, performance capture, time-lapse, morphing, Augmented Reality and the use of avatars in human-computer communication has become increasingly popular, cheap and simple.
\end{abstract}

Key words: computer graphics, computer animation, performance capture, human-computer communication

\section{Introduction}

In the past, the market for (user) applications software was monopolized by a few leading and well-known companies. The market valued cult products, and the data recording formats associated with them. The last decade has seen the spread of the use of cloud computing, which allows end users to run computer programs online in a web (internet) browser. Previously, all programs had to be installed on one's own computer and were run from the desktop. Since the prices of user programs of that time were a result of the laws of supply and demand, they differed significantly from the cost of production. It turned out that for entry-level companies it paid to provide them on the cloud for free to a certain extent or even completely. The phenomenon of free software, and the movement within it known as Open Source, has been around for a long time of course. From the year 2010 sites with online applications in the cloud started to appear en masse, and so it was in the field of computer graphics. For example, competitors to the cult program Microsoft Vision (acquired in 2000 
from the Shapeware Corporation) appeared in the form of online programs such as Gliffy, Draw.io, Lucid chart and dozens of others [1].

In the last five years such has been the story of the new technologies of human-computer communication: Kinect and Leap Motion. They allow the control of a program in real-time by using body movements, for example by pointing a finger rather than moving a computer mouse. Many computer technologies are descendants of much earlier technologies, for ex-ample, in films, photography and animation. Similiarly older computer technologies influence the development of ones that are currently popular. An example is motion capture technology, which used to be expensive and require recording studios and the installation of markers on moving actors. Today it is very cheap and accessible in games consoles and computers in the form of the technologies Kinect or Leap Motion.

This paper presents an overview of some selected applications of new computer graphics and computer animation technologies. Also discussed are examples of software invaluable for fast and cost-effective creation of interesting applications.

\section{Rotoskop stop-motion animation technology}

Stop-motion animation is one technology for drawing, imaging and computer animation. It involves displaying images at specific intervals, e.g. 24 frames per second. The history of animation dates back to the end of the nineteenth century and its progenitor was Léon Gaumont (1864-1946), who was awarded a French patent for stop-motion animation in 1900. As with animations, so with film and digital video. An example of Time-lapse stop-motion animation is a time-lapse movie composed of many images made with, for example, a camera. The resulting film is very impressive in that it allows one to view phenomena imperceptible to the human eye because of their very slow pace. In this way one can present an animation of the growth of a plant taking place at a faster rate than in reality [8] [21].

An interesting method of stop-motion animation is rotoskop, in which movie frames with live actors are substituted for ones that are manually drawn by a cartoonist. A new use of rotoskop animation technology is a first fulllength animated film which is dedicated to the Dutch painter Vincent van Gogh. 


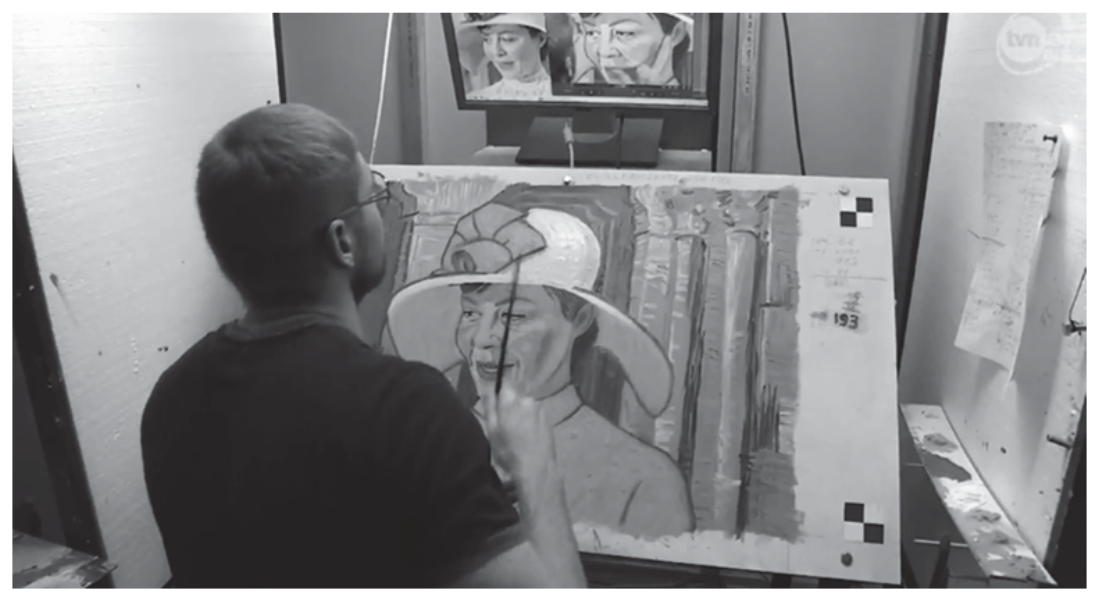

Figure 1. Movie frame with the image of an actor hand-painted with oil paints on canvas [13]

The director and designer - Dorota Kobiela - is Polish. The lead animation producer is Hugh Welchman, who won an Oscar for best short animated film with „Peter and the Wolf” in 2008. The screenplay was a collaboration by Kobiela and Jack Dehnel, a Polish poet, translator and painter. „Twój Vincent” („Loving Vincent”) will be an 80 -minute film about the life of van Gogh. It will consist of more than 56,000 frames - photographs of oil-oncanvas paintings done in the Expressionist style characteristic of the artist Figure 1. About 100 Polish painters, working in studios in Gdansk, Wroclaw and Great Britain, were invited to participate by the producers. Together they created more than 43,200 images, making 12 images for each second of film. About 120 original paintings by van Gogh feature in the film [13].

\section{Computer applications that simulate the knowledge and skills of a painter}

Research is being carried out into automating the process of painting pictures. An inter-national team consisting of world-class scientists, engineers, programmers and art historians decided to take a challenge and see if a program could be developed to enable a computer to simulate the skills of one of the greatest and most innovative artists of all time - Rembrandt - and control a 3D printer [2]. All of Rembrandt's paintings were scanned using a 3D scanner in order to analyse his style and to assess the thicknesses of the layers of paint he applied. After the scanning of 346 of the artist's works and their analysis by a team of researchers, software with the knowledge and skills of the brilliant 
painter was developed. A significant par of Rembrandt's work is portraits, so the researchers focused on those in particular Figure 2.

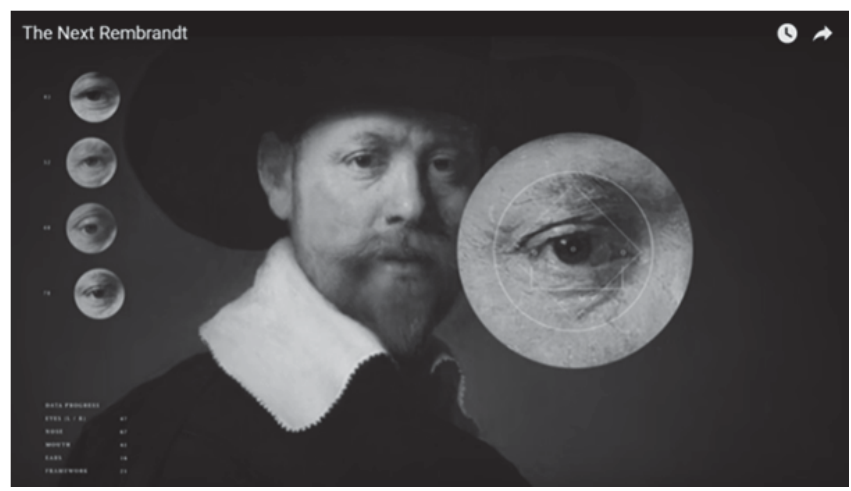

Figure 2. 3D scan and analysis of the painter's works [2]

Once the software was developed, a made-up portrait of the Dutch painter was produced with a 3D printer based on a database containing information about his original portraiture. The results of such research can be used to create accurate reproductions, and contribute to making the achievements of the great painter more widely known. The software created by the researchers could also be used for commercial purposes, and then we would all be able to order our own portrait created by a virtual seventeenth century Rembrandt for our homes.

\section{Animation techniques based on motion capture}

The first type of stop-motion animation was motion capture technology, the capture of movements of selected points of three dimensional real objects e.g. the human body. An actor puts on a vest with markers (sensors) attached, the movements of which are captured with a camera onto film frames Figure 3. Markers are also attached to the face, in order to record facial expressions. The positions of these markers on individual animation frames helps graphic designers to create very lifelike motion-animated characters. Studio SoInteractive is an example of a company involved in animation design based on the technique of motion capture [9] [28]. 


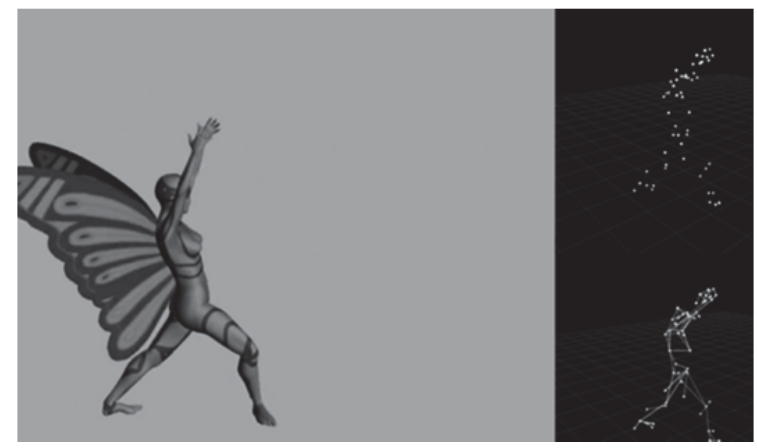

Figure 3. The use of markers to record the movements of points on 3D objects [9]

In 2010 there was a revolutionary change in the animation technique of motion capture because two new technologies appeared on the market. Microsoft brought out the technology Kinect [19] and a similiar solution appeared from the Leap Motion company [15]. A snap-in to a computer with two cameras registers the movement of $3 \mathrm{D}$ objects. The application of these new technologies no longer requires the use of markers because the computer programs can detect characteristic points of the observed objects, for example moving fingers or hands Figure 4.

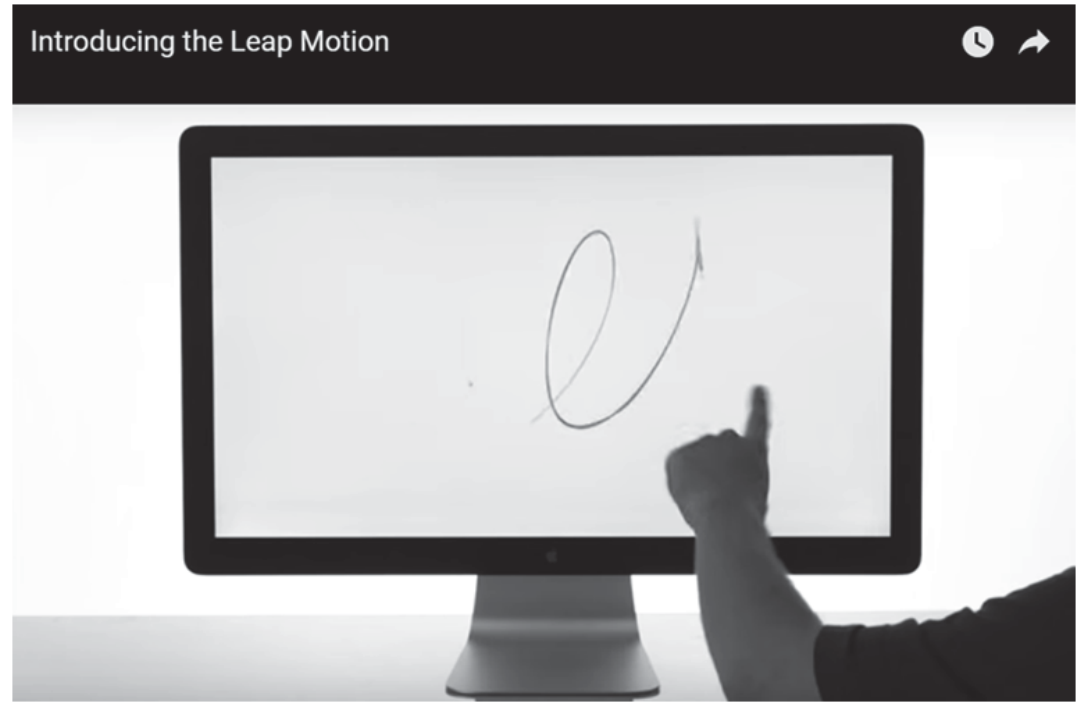

Figure 4. Capturing the movement of real objects [14] 


\section{Inbetweening animation technology}

Morphing technology is the simplest example of automatic animation also known as inbetweening. It relies on software to generate graphics for intermediate frames. Thus we enter images, for example of a face, for two frames which are called key frames. For both raster images the program identifies characteristic points, such as the corners of the eyes, and on this basis determines the position of these points in the intermediate frames using a calculation algorithm. Then the filling in between the points is rendered with fragments of raster graphics derived from both key frames [16].

An example of the use of morphing would be an animation that shows a person's face changing over the course of a lifetime. Photographs of the face taken at various life stages are entered into the morphing program. The applications software generates intermediate frames creating an animation of the person's face [20].

Another example of programs with software that generates animation frames is provided by applications for creating avatars [22] [3]. An avatar can be animated from a drawing of any object, or from a 2D photograph of a human. Many companies providing software and services that produce various types of avatars have appeared on the market [31]. Avatars can consist of only part of an object, for example a head [5], or the whole object [17]. One of the most popular programs for animation of the head, along with facial expressions and lip movements, is the program CrazyTalk, currently in version 8 , from the company Reallusion [25].

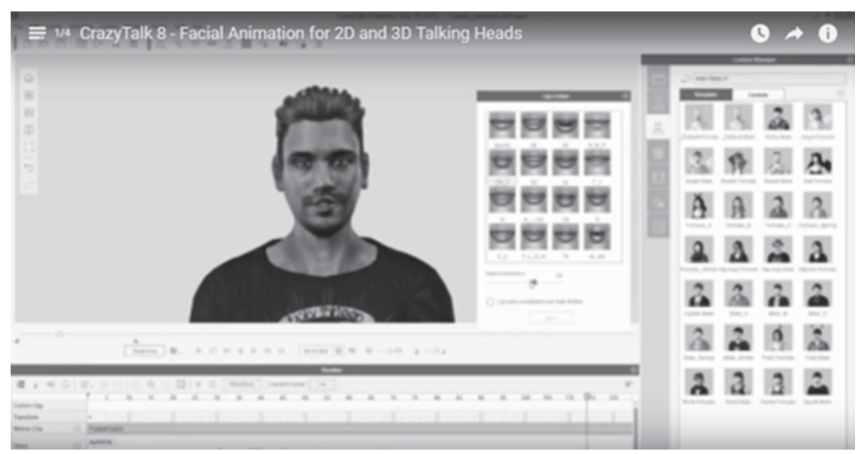

Figure 5. The CrazyTalk application site, selecting an avatar's teeth [25]

This program enables the animation of a 3D avatar head from photographs or 2D drawings. During the automatic animation facial expression and lip movements are added, which can articulate words that are requested with a text or audio file (Figure 5). Such an avatar is con-trolled on the one hand 
with a dynamic behaviour program, and on the other with a script for its speech.

There are also free sites, deserving of wider use, on which it is possible to design video animations for your specific requirements, without any need for programming knowledge [32] [7] [27] [6].

\section{Controlling applications in real-time using body motion capture - performance capture}

Motion capture technology enables the use of natural movements of selected points of the body in animation, which indicate where the same points will be located in animated shapes at a given moment in time. The current development of this technology is referred to as performance (facial motion) capture and involves not only mapping body movements, but also scanning human facial expressions [10]. Performance computing along with the very cheap technologies Kinect or Leap Motion allow the capture and use of whole-body movements to control computer programs in real time Figure 6.

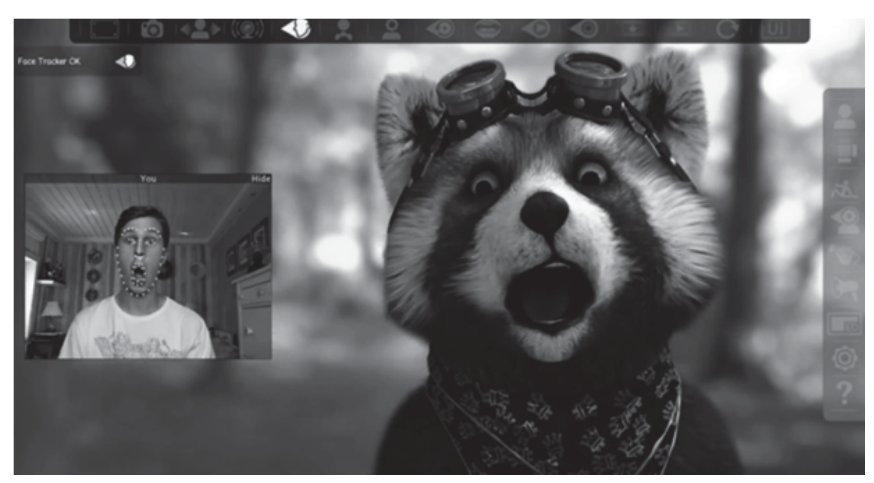

Figure 6. Scanning facial expressions and transposing them on the face of an avatar [10]

A company called Reallusion, which has been known for a long time for its program CrazyTalk, offers among other things a program called iClone, which allows 3D animation of a whole figure along with the head in Kinect and Kinect Mocap plug-in technology [26]. 


\section{Linking computer graphics with real world images - augmented reality}

Virtual Reality is an example of the use of 3D computer graphics and has become increasingly popular. Its popularity, even on 2D monitors, stems from the potential of creating increasingly realistic $2 \mathrm{D}$ images with it, which are deceptively reminiscent of real world images viewed through human eyes. Of course stereoscopic viewing of 3D objects or watching 2D animations in 3D technology makes an even greater impression. An interesting example is the reconstruction of Jan Matejko's painting „Battle of Grunwald” with stereoscopic technology Figure 7. This project was commissioned by the National Museum in Warsaw and created by the studio Platige Image [23] [12].

An interesting idea is to combine two views - from the real world and the virtual one - and view them on the screen at the same time. Such a solution is called Augmented Reality (AR). A Virtual Reality (VR) view can also be combined with another one to create an Augmented Virtuality (AV). The synchronisation of these two worlds can be performed using point markers or by reference to a geographic position. An example of the use of augmented reality are Microsoft Hololens glasses [18], which are specially tailored to this kind of visualisation. Computer graphics can become complementary to the real world with this technology. For example, with Hololens glasses a lineman sees the repaired part of a sewerage system and at the same time carries out a conversation with an expert on Skype (seeing it on a virtual screen Figure 7, and also sees a graphic which shows which components can be loosened and with which movements [18].

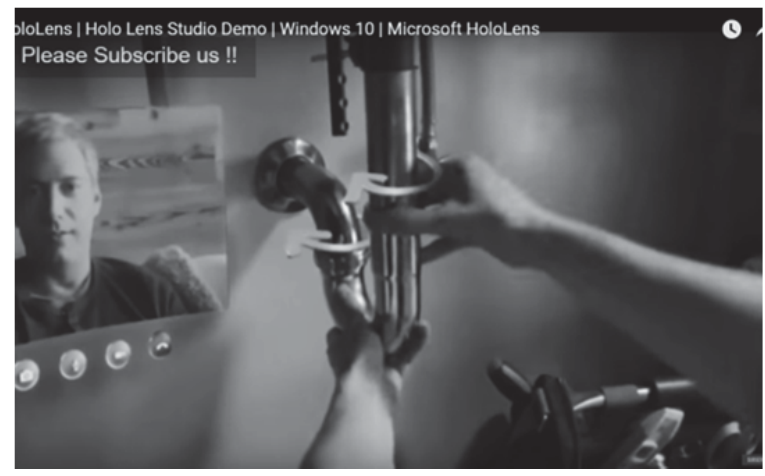

Figure 7. With Hololens glasses a lineman sees a sewage system, virtual computer screen and an infographic [18] 
In museums virtual avatars of historical figures may appear as guides. Strong interest in using augmented reality is shown in web and traditional marketing, for example to create a virtual fitting room [4].

\section{Facial recognition and identification of facial expressions}

The development of these technologies in recent times is linked not only with animation, but also with facial recognition. The programmers of Facebook have built software which recognises faces with $97.25 \%$ accuracy, which approaches the capabilities of a human. The program works regardless of prevailing light conditions or the angle of inclination of the head [30].

In 2016, Amazon filed an application for a patent that describes a shopping method which will make use of facial recognition and facial expressions to authorise transactions [24]. After accepting a transaction in an online store, the site launches a camera application and performs a two-step verification process. The first, of course, is facial recognition. At the second stage, the site launches a camera that records facial expressions. For example, it could request that the user of the site winks with the right eye or smiles.

In 2013, at the Techfest conference, Microsoft presented the results of research carried out in the area of recognition of facial expressions and voice tone [29]. The aim of the project ,turning monolingual speaker into multilingual" was, in the first instance, the simulation of a human face speaking a native language. Further research led to the possibility of real-time simulation of the face of the same human speaking 26 languages.

A team of researchers from the University of Washington designed software which analysed thousands of photos posted on the Internet from the point of view of the incremental changes in the appearance of a human face in the course of a lifetime, and on this basis is able to estimate the appearance of a human face in any period of life. The program is so powerful that it can picture a face at any given time in a human life even in different poses, with different expressions and in unfavourable light. It is particularly difficult to predict how a child will look like in later years of life, because the shape of a face changes dramatically with age. This time however, the researchers say, the program is so accurate that it can calculate each dimension of the head and shape of the face, taking into account different age groups; it generates the target portrait on that basis. The study was financed by Google and Intel Corporation [11].

\section{Conclusions}

In the examples given of computer graphics and animation the appeal of the applications software and its increasing capabilities are apparent. There is also 
potential for further development. There is nothing to prevent film frames with actors from the van Gogh film being transformed by computer into frames of animated film using the program that was created for the works of Rembrandt. The automation of film production with computer applications is a major technological achievement. Museums will become more attractive and valuable exhibits more popular as, with low costs and seamless technologies, it will be possible to display faithful copies of those exhibits anywhere. Graphics programs that permit the analysis of photographs of the face, eyes or hands for the purpose of medical diagnosis will emerge.

Currently, graphics applications software is designed for an even wider range of uses. The tools are better adapted to the needs of artists and more convenient to use. Continuous progress in the field means that it is necessary to follow the innovations and potential of soft-ware from different producers, and not be tied to just one firm as in the past. The examples of the technologies Kinect and Leap Motion are evidence of the scale of change of the last five years. Performance computing and the very cheap technologies Kinect and Leap Motion al-low the capture and use of whole body movements for real-time control of computer pro-grams. The use of graphic applications software with motion capture technology, performance capture, time-lapse, morphing, Augmented Reality or the use of avatars in human-computer communication is becoming increasingly popular, cheap and simple.

\section{References}

1. AN Srikanth, 2015, Visio Alternatives: 10 Best Diagramming Software, http://beebom.com/visio-alternatives/

2. Brown M., 2016, 'New Rembrandt' to be unveiled in Amsterdam, https://www.theguardian.com/artanddesign/2016/apr/05/new-rembrandt-to-beunveiled-in-amsterdam

3. Endicott M. L., 2014, 100 Best Talking Avatar Videos, http://metaguide.com/videography/100-best-talking-avatar-videos

4. Filutowicz Z., Przybyszewski K., Sowa G., Paszkowski J., 2012, Techniques of digital photography and computer graphics visualization e-business, Studies \& Proceedings of Polish Association for Knowledge Management" Tom 62, Nr 1, pp17-31

5. Fleming B, Dobbs D., 2002 Animating Facial Features and Expressions Paperback, Helion, 2002

6. FreeMake, 2016, How to Make a Cartoon Yourself: Top 7 Animated Video Makers Compared, http://www.freemake.com/blog/5-best-sites-to-make-animatedvideo-trouble-free/

7. GoAnimate, 2016, Make Professional Animated Videos GoAnimate, https://goanimate.com/ 
8. Google, 2016, Google Earth Engine Timelapse, https://earthengine.google.com/timelapse/

9. Hildebrand K., 2012, Polygamia.pl: How does the motion capture studio? https://www.youtube.com/watch?v=R-N-BpeHm28

10. Holotech Studios, 2016, FaceRig, https://facerig.com/

11. Kemelmacher-Shlizerman I., Suwajanakorn S., Seitz S. M., 2014, IlluminationAware Age Progression, http://grail.cs.washington.edu/aging/

12. Kidziński R., Bagiński T., 2010, Jan Matejko's "Battle of Grunwald" in 3D, http://www.playpoland.org.uk//index.php?name=movies_more\&oId=80

13. Kobiela D., Welchman H., 2016, Loving Vincent, http://lovingvincent.com/

14. Leap Motion, 2012, Introducing the Leap Motion, https://www.youtube.com/watch? $\mathrm{v}=$ d6KuiuteIA

15. Leap Motion, 2016, Reach into new worlds, https:/www.leapmotion.com/

16. Lindquist T., 2011, Cat-to-dog morph, https:/www.youtube.com/watch?v=VV807_NqGto

17. Maestri G., 2000, Character Animation, Helion

18. Microsoft HoloLens, 2015, Mixed reality: Your world is the canvas, https://www.microsoft.com/microsoft-hololens/en-us

19. Microsoft Kinect, 2016, Meet Kinect for Windows, https:/www.developer.microsoft.com/en-us/windows/kinect

20. MovieTrailersNET, 2012, Morphing 33 years in 60 seconds, https://www.youtube.com/watch?v=1AE-gUuu814

21. National Geographic, 2014, Time-Lapse: Watch Flowers Bloom Before Your Eyes, https://www.youtube.com/watch?v=LjCzPp-MK48

22. Plantec P., Kurzwell R. M., 2003Virtual Humans: A Build-It-Yourself Kit, Complete with Software and Step-by-Step Instructions, New York: AMACOM

23. Platige Image, 2016, Platige to Artistic Conglomerate, http://www.platige.com/pl/

24. Rao L., 2016, Amazon Is Trying to Patent Paying With a Selfie, http://fortune.com/2016/03/14/amazon-patent-selfie/

25. Reallusion, 2016, CrazyTalk, http://www.reallusion.com/crazytalk/default.html

26. Reallusion, 2016, iClone, http://www.reallusion.com/iclone/default.html

27. Skavish D., That's how the idea for Animatron was born, https://editor.animatron.com/

28. SoInteractive, 2012, The film presents the possibilities of studying SoInteractive confirms the good condition of the Polish sector of motion capture, http://www.gry-online.pl/S013.asp?ID=68466

29. Soong F., 2012, Turning a Monolingual Speaker Into Multi-Lingual Speaker, http://research.microsoft.com/en-us/projects/mixedlangtts/

30. TaigmanY., Yang M., Ranzato M. A., Wolf L., 2014, DeepFace: Closing the Gap to Human-Level Performance in Face Verification, 
https://research.fb.com/publications/deepface-closing-the-gap-to-human-levelperformance-in-face-verification/

31. van Lun E., 2016, Virtual Agents / Chatbots Directory, https://www.chatbots.org/

32. Wideo.co, 2016, Create videos easily for your marketing campaigns, http://get.wideo.co/en/ 